\title{
Corrigendum
}

\section{Dendritic spine loss and neurodegeneration is rescued by Rab11 in models of Huntington's disease}

\author{
P Richards, C Didszun, S Campesan, A Simpson, B Horley, KW Young, P Glynn, K Cain, CP Kyriacou, F Giorgini and P Nicotera
}

Cell Death and Differentiation (2011) 18, 914; doi:10.1038/cdd.2011.15

Correction to: Cell Death and Differentiation (2011) 18, 191-200; doi:10.1038/cdd.2010.127; published online 19 November 2010

Since the publication of this article, the authors noticed that the 'DZNE address' was inserted into the second paragraph of the introduction. The corrected paragraph is shown below.

Although protein aggregates are a consistent feature of many neurodegenerative diseases, ${ }^{7}$ their role in the degenerative process is unclear. Although several studies have found aggregates to be associated with cell death, ${ }^{8-10}$ others have found no correlation between aggregation of mutant htt and cellular toxicity. ${ }^{11-13} \mathrm{~A}$ provocative study has found that the presence of inclusion bodies containing mutant htt may actually predict lower risk of cell death in primary neurons. ${ }^{14}$ Indeed, it has been suggested that htt aggregates may represent a molecular sink for soluble toxic htt forms. ${ }^{11,14}$ Recent work indicates that soluble oligomers or 'microaggregates' of mutant htt, which may represent one of the toxic species of misfolded htt critical for HD pathology, form in a polyQ-dependent manner both in vitro and in vivo. ${ }^{15}$ Such soluble aggregate species precede symptoms in HD mice and may contribute to pathology in this model system. ${ }^{16}$

The authors would like to apologize for any inconvenience this may have caused. 